\title{
FREE PRODUCTS OF VON NEUMANN ALGEBRAS(1)
}

\author{
BY
}

\section{WAI-MEE CHING}

ABSTRACT. A new method of constructing factors of type $\mathrm{II}_{1}$, called free product, is introduced. It is a generalization of the group construction of factors of type II ${ }_{1}$ when the given group is a free product of two groups. If $A_{1}$ and $A_{2}$ are two von Neumann algebras with separating cyclic trace vectors and ortho-unitary bases, then the free product $A_{1} * A_{2}$ of $A_{1}$ and $A_{2}$ is a factor of type II without property $\Gamma$.

1. Introduction. In the study of von Neumann algebras, factors of type $\mathrm{II}_{1}$ have been constructed by the so-called measure-construction, the group-construction, and the infinite tensor product (see Murray and von Neumann [10], [11], von Neumann [20]). A more general construction, called crossed product, which includes the measure-construction and the group-construction as special cases has been studied by Nakamura and Takeda [12], Suzuki [17], Turumaru [19], and Ching [3]. All hyperfinite factors of type $\mathrm{II}_{1}$ are isomorphic to each other (Murray and von Neumann [11]), and a hyperfinite factor of type $\mathrm{II}_{1}$ can be constructed by the measure-construction, the group-construction, or the infinite tensor product. This indicates that factors of type $\mathrm{II}_{1}$ produced from quite different methods may actually be the same, i.e. isomorphic to each other. On the other hand, all nonhyperfinite factors of type $\mathrm{II}_{1}$ so far discovered and classified, are constructed by the group-construction (see Ching [3], [4], Dixmier and Lance [5], McDuff [8], [9], Murray and von Neumann [11], Sakai [14], [15], Schwartz [16], Zeller-Meier [21]). The question that arises then is whether all factors of type $\mathrm{II}_{1}$ can be produced by the group construction; or put in another way, is any factor of type $\mathrm{II}_{1}$ isomorphic to $\mathfrak{Q}(G)$ for some discrete group $G$, where $\mathbb{Q}(G)$ is the von Neumann algebra generated by the regular representation of $G$. This paper resulted from an attempt to give the above question hopefully a negative answer. We introduce a new method to construct a factor of type $\mathrm{II}_{1}$, called free product, which is modeled after the free product of groups. It is, in fact, a generalization of the group-construction, with the given group being a free product of two groups. For example, we would see that the free product

Received by the editors February 29, 1972.

AMS (MOS) subject classifications (1970). Primary 46L10.

Key words and phrases. von Neumann algebra, group construction factor of type $\mathrm{II}_{1}$, free product, property $\Gamma$.

(1) This research was supported by National Science Foundation grant GN-28517. 
$\mathbb{Q}(Z) * \mathbb{Q}(Z)$ is isomorphic to $\mathbb{Q}\left(\Phi_{2}\right)$, where $Z$ is the integer group and $\Phi_{2}$ is the free group with two generators. We shall show that a free product of two finite von Neumann algebras with ortho-unitary bases, one of linear dimension at least two, the other of dimension at least three, is always a nonhyperfinite factor without property $\Gamma$. Although the motivation of this paper is to construct a factor of type $\mathrm{II}_{1}$ nonisomorphic to any group-von Neumann algebra $\mathfrak{A}(G)$, we are not able, at present, to show that such a factor actually exists. For instance, it is not known whether $M_{2} * M_{3}$, the free product of the 2 by 2 matrix algebra and the 3 by 3 matrix algebra, is isomorphic to $\mathfrak{Q}\left(\Phi_{2}\right)$.

Another unsolved problem concerning von Neumann algebras is whether a factor of type $\mathrm{II}_{1}$ necessarily has an outer automorphism. All factors of type $\mathrm{I}_{n}$ (i.e. $n \times n$ matrix algebra) do not have any outer automorphism (Dixmier [5, Proposition 4, $\mathrm{p}$. 255]). Recently, Takesaki [18] proved that a factor of type III always has an outer automorphism. Any locally compact group can be represented as a group of outer automorphisms of the hyperfinite factor of type $\mathrm{II}_{1}$ (Blattner [2]). Behncke [1] showed that this result is still true if the hyperfinite factor is replaced by $\mathbb{Q}\left(\Phi_{2}\right)$. We shall see that these known methods of finding an outer automorphism of a factor of type $\mathrm{II}_{1}$ cannot yield any outer automorphism of $M_{2} * M_{3}$ for us. However, the task to determine all outer automorphisms of $M_{2} * M_{3}$ (probably none) must be left to further study of free products of von Neumann algebras.

All Hilbert spaces in this paper are complex and separable and all groups in this paper are countable. The identity operator in all von Neumann algebras is denoted by 1 , and we shall call a scalar multiple of identity simply a scalar.

The author would like to thank Professor C. Heinrich for some helpful discus sions.

2. Construction of the free product. Let $A_{i}$ be a von Neumann algebra on Hilbert space $H_{i}$ with a cyclic separating trace vector $\xi_{i}$ (of norm 1 ), $i=1,2$. Let $A=A_{1} \otimes A_{2}$ be the algebraic tensor product of $A_{1}$ and $A_{2}$. Let $A^{n}=A \otimes \ldots$ $\otimes A$ be the $n$th algebraic tensor power of $A$. Let $\tilde{A}=\sum \bigotimes_{n=1}^{\infty} A^{n}$ be the tensor algebra of $A$ without the summand of the field of constants. A general element of $\widetilde{A}$ is of the form

$$
\sum_{k=1}^{m} \sum_{i=1}^{n} x_{1, i} \otimes y_{1, i} \otimes \cdots \otimes x_{k, i} \otimes y_{k, i}
$$

where $x_{j, i} \otimes y_{j, i} \in A_{1} \otimes A_{2}, 1 \leq i \leq n_{k}, 1 \leq j \leq k, 1 \leq k \leq m$. Let $I$ be the ideal of $\tilde{A}$ generated by elements $\left\{x_{1} \otimes y_{1} \otimes \cdots \otimes x_{i} \otimes 1 \otimes x_{i+1} \otimes y_{i+1} \otimes\right.$ $\cdots \otimes x_{n} \otimes y_{n}-x_{1} \otimes y_{1} \otimes \cdots \otimes x_{i-1} \otimes y_{i-1} \otimes x_{i} x_{i+1} \otimes y_{i+1} \otimes \cdots \otimes x_{n}$ $\otimes y_{n}, x_{1}^{\prime} \otimes y_{1}^{\prime} \otimes \cdots \otimes x_{i-1}^{\prime} \otimes y_{i-1}^{\prime} \otimes 1 \otimes y_{i}^{\prime} \otimes \cdots \otimes x_{m}^{\prime} \otimes y_{m}^{\prime}-x_{1}^{\prime} \otimes$ 
$\left.y_{1}^{\prime} \otimes \ldots \otimes x_{i-1}^{\prime} \otimes y_{i-1}^{\prime} y_{i}^{\prime} \otimes x_{i+1}^{\prime} \otimes y_{i+1}^{\prime} \otimes \ldots \otimes x_{m}^{\prime} \otimes y_{m}^{\prime}\right\}$. Let $\bar{A}=\tilde{A} / I$ be the quotient algebra. An element $a$ in $\tilde{A}$ of the form $x_{1} \otimes y_{1} \otimes \cdots \otimes x_{n} \otimes y_{n}$ is called a monomial, which we shall write simply $x_{1} y_{1} \cdots x_{n} y_{n}$ hereafter. $n$ is called the length of the monomial, and is denoted by $\rho(a)=n$. Every element of $\tilde{A}$ is a sum of monomials.

Any element $x$ of $A_{i}$ can be written as $x=c 1+x^{\prime}$, where $c=\operatorname{tr}(x)=\left(x \xi_{i} \mid \xi_{i}\right)$, and $x^{\prime}=x-c 1$ (hence $\left.\operatorname{tr}\left(x^{\prime}\right)=0\right), i=1,2$. A monomial $x_{1} y_{1} \cdots x_{n} y_{n}$ is said to be irreducible if $\operatorname{tr}\left(x_{i}\right)=0$ and $\operatorname{tr}\left(y_{i}\right)=0$ for all $i$ with the possible exception of $x_{1}$ and $y_{n}$, each of which is either of trace zero or a scalar. Every monomial $x_{1} y_{1}$ $\cdots x_{n} y_{n}$ (and hence every element of $\tilde{A}$ ) is equivalent to a finite sum $\overline{x_{1} y_{1} \cdots x_{n} y_{n}}$, unique up to a rearrangement of summation, of irreducible monomials each having length no more than $n$. The unique sum $\overline{x_{1} y_{1} \cdots x_{n} y_{n}}$ of irreducible monomials is called the canonical form of $x_{1} y_{1} \cdots x_{n} y_{n}$. This can be readily proved by induction.

Lemma 1. There exists a rewriting process to reduce every monomial $x_{1} y_{1}$ $\cdots x_{n} y_{n}$ into a unique canonical form $\overline{x_{1} y_{1} \cdots x_{n} y_{n}}$.

Proof. It is trivially true for the case $n=1$. Suppose that $\overline{x_{1} y_{1} \cdots x_{k} y_{k}}$ has been defined for $k<n$. Let $x_{1} y_{1} \cdots x_{n} y_{n}$ be given. Suppose

$$
\overline{x_{1} y_{1} \cdots x_{n-1} y_{n-1}}=\sum_{k=1}^{n-1} \sum_{i=1}^{n} x_{1, i} y_{1, i} \cdots x_{k, i} y_{k, i} \text {, }
$$

where $x_{1, i} y_{1, i} \cdots x_{k, i} y_{k, i}$ is an irreducible monomial, $1 \leq i \leq n_{k}, 1 \leq k \leq m$.

Define

$$
\overline{x_{1} y_{1} \cdots x_{n} y_{n}}=\sum_{k=1}^{n-1} \sum_{i=1}^{n} \overline{x_{1, i} y_{1, i} \cdots x_{k, i} y_{k, i} x_{n} y_{n}},
$$

where each $\overline{x_{1, i} y_{1, i} \cdots x_{k-1, i} y_{k-1, i}{ }^{x} y_{n}}$ is defined as the following: first of all,

$$
\begin{aligned}
\overline{x_{1, i} y_{1, i} \cdots x_{k-1, i} y_{k-1, i} x_{n} y_{n}} \\
\quad=\operatorname{tr}\left(y_{n}\right) \overline{x_{1, i} y_{1, i} \cdots x_{k-1, i} y_{k-1, i} x_{n}^{1}}+\overline{x_{1, i} y_{1, i} x_{k-1, i} y_{k-1, i} x_{n} y_{n},}
\end{aligned}
$$

where $y_{n}^{\prime}=y_{n}-\operatorname{tr}\left(y_{n}\right) 1$. Hence, we can assume either $\operatorname{tr}\left(y_{n}\right)=0$ or $y_{n}=c 1$. Now, (i) if $\operatorname{tr}\left(x_{n}\right)=0$ and $\operatorname{tr}\left(y_{k-1, i}\right)=0$, then

$$
\overline{x_{1, i} y_{1, i} \cdots x_{k-1, i} y_{k-1, i} x_{n} y_{n}}=x_{1, i} y_{1, i} \cdots x_{k-1, i} y_{k-1, i} x_{n} y_{n} ;
$$

(ii) if $x_{n}=c l$ is a scalar, then

$$
x_{1, i} y_{1, i} \cdots x_{k-1, i} y_{k-1, i} x_{n} y_{n}=c x_{1, i} y_{1, i} \cdots x_{k-1, i}\left(y_{k-1, i} y_{n}\right) \text {, }
$$


which is already defined by our assumption; (iii) if $y_{n-1, i}=c 1$, then

$$
\overline{x_{1, i} y_{1, i} \cdots x_{k-1, i} y_{k-1, i} x_{n} y_{n}}=c \overline{x_{1, i} y_{1, i} \cdots\left(x_{k-1, i} x_{n}\right) y_{n}} \text {, }
$$

which is already defined. For general $x_{n}, x_{n}=\operatorname{tr}\left(x_{n}\right) 1+x_{n}^{\prime}$, with $\operatorname{tr}\left(x_{n}^{\prime}\right)=0$. And we define

$$
\begin{aligned}
& \overline{x_{1, i} y_{1, i} \cdots \bar{x}_{k-1, i} y_{k-1, i} x_{n} y_{n}} \\
& =\operatorname{tr}\left(x_{n} \overline{x_{1, i} y_{1, i} x_{k-1, i}\left(y_{k-1, i} y_{n}\right)}+\overline{x_{1, i} y_{1, i} \cdots x_{k-1, i} y_{k-1, i} x_{n}^{\prime} y_{n}} .\right.
\end{aligned}
$$

This proves the lemma.

For every element $a=\Sigma a_{i}$ in $\tilde{A}$, where the $a_{i}$ 's are monomials, let $\bar{a}=$ $\Sigma \bar{a}_{i} \cdot \bar{a}$ is a unique representative of the equivalence class of $a$ in $\bar{A}=\widetilde{A} / I$. So, we can regard $\bar{a}$ as the element in $\bar{A}$ it represents. Define $c \cdot \bar{a}=\overline{c \cdot a}$ for complex number $c, \bar{a} \cdot \bar{b}=\overline{a \cdot b}, \bar{a}+\bar{b}=\overline{a+b}$, where $a \cdot b$ is the multiplication in $\tilde{A}$, in the case of the two monomials

$$
\left(x_{1} y_{1} \cdots x_{n} y_{n}\right) \cdot\left(x_{1}^{\prime} y_{1}^{\prime} \cdots x_{m}^{\prime} y_{m}^{\prime}\right)=x_{1} y_{1} \cdots x_{n} y_{n} x_{1}^{\prime} y_{1}^{\prime} \cdots x_{m}^{\prime} y_{m}^{\prime},
$$

and in the case of the two general elements it is defined by the linear extension of the above operation. It is easy to see that $\bar{A}$ is the same complex associative algebra as defined by quotient $\tilde{A} / I$. We can regard $A$ as consisting of linear combinations of irreducible monomials. Furthermore, we define $\left(x_{1} y_{1} \cdots x_{n} y_{n}\right)^{*}=$ $\overline{1 y_{n}^{*} x_{n}^{*} y_{n-1}^{*} \cdots x_{2}^{*} y_{1}^{*} x_{1}^{*} 1}$ for an irreducible monomial $x_{1} y_{1} \cdots x_{n} y_{n}$. Extending the conjugate linearly to all elements of $\bar{A}, *$ is an involution of $\bar{A}$ such that $(c \cdot a)^{*}=$ $\bar{c} \cdot a^{*}$, and $(a b)^{*}=b^{*} a^{*}$ for complex number $c$, and $a, b \in \bar{A}$.

Define a function $f$ by $f\left(x_{1} y_{1} \cdots x_{n} y_{n}\right)=0$ for an irreducible monomial $x_{1} y_{1}$ $\cdots x_{n} y_{n}$ in $A$, except for the case $n=1$ and $x_{1} y_{1}=c 1$, a scalar, for which we define $f\left(x_{1} y_{1}\right)=c$. Extend this function linearly to all elements of $A$. Note that for a monomial of length one (not necessarily irreducible), $f\left(x_{1} y_{1}\right)=\operatorname{tr}\left(x_{1}\right) \operatorname{tr}\left(y_{1}\right)$, where $\operatorname{tr}\left(x_{1}\right)$ and $\operatorname{tr}\left(y_{1}\right)$ are the traces of $A_{1}$ and $A_{2}$ respectively. We have

Lemma 2. The linear functional / defined on $\bar{A}$ bas the following properties:

(a) $f(a b)=f(b a)$ for any $a, b \in \bar{A}$,

(b) $f\left(a^{*} a\right)>0$ for all nonzero element $a$ in $\bar{A}$,

(c) $f\left(a^{*}\right)=\overline{f(a)}$ for any $a \in \bar{A}$.

Proof of (a). It is clear that we only need to prove (a) for $a=x_{1} y_{1} \cdots x_{n} y_{n}$, and $b=s_{1} t_{1} \cdots s_{m}{ }_{m}$ being two irreducible monomials in $\bar{A}$.

Case 1 . Neither $y_{n}$ nor $s_{1}$ is a scalar. Hence

$$
a b=x_{1} y_{1} \cdots x_{n} y_{n} s_{1} t_{1} \cdots s_{m} t_{m} \text {, and } f(a b)=0 .
$$

(i) If neither $x_{1}$ nor $t_{m}$ is a scalar, then 
(1)

$$
b a=s_{1} t_{1} \cdots s_{m} t_{m} x_{1} y_{1} \cdots x_{n} y_{n} \text {, and } f(b a)=0 .
$$

(ii) If $x_{1}$ is a scalar, but $t_{m}$ is not, then

$$
\begin{aligned}
f(b a) & =\operatorname{tr}\left(x_{1}\right) f \overline{\left(s_{1} t_{1} \cdots s_{m}\left(t_{m} y_{1}\right) x_{2} y_{2}\right.} \overline{\left.x_{n} y_{n}\right)} \\
& =\operatorname{tr}\left(x_{1}\right) \operatorname{tr}\left(t_{m} y_{1}\right) f \overline{\left(s_{1} t_{1} \cdots s_{m-1} t_{m-1}\left(s_{m} x_{2}\right) y_{2} \cdots x_{n} y_{n}\right)}
\end{aligned}
$$

(2) $\quad f(b a)=\operatorname{tr}\left(x_{1}\right) \operatorname{tr}\left(t_{m} y_{1}\right) \operatorname{tr}\left(s_{m} x_{2}\right) \cdots \operatorname{tr}\left(t_{m-1+1} x_{i+1}\right) \cdots \operatorname{tr}\left(t_{1} y_{n}\right) \operatorname{tr}\left(s_{1}\right)=0$, in case $m=n$;

$$
f(b a)=\operatorname{tr}\left(x_{1}\right) \operatorname{tr}\left(t_{m} y_{1}\right) \operatorname{tr}\left(s_{m} x_{2}\right) \cdots \operatorname{tr}\left(t_{1} y_{n-1}\right) \operatorname{tr}\left(s_{1} x_{n}\right) \operatorname{tr}\left(y_{n}\right)=0,
$$

in case $n=m+1$. In all other cases, $b a$ is a sum of irreducible monomials all of length at least two, hence $f(b a)=0$.

(iii) If $t_{m}$ is a scalar, but $x_{1}$ is not, then

$$
\begin{array}{r}
f(b a)=\operatorname{tr}\left(t_{m}\right) f\left(\overline{\left.s_{1} t_{1} \cdots s_{m-1} t_{m-1}\left(s_{m} x_{1}\right) y_{1} \cdots x_{n} y_{n}\right)}\right. \\
=\operatorname{tr}\left(t_{m}\right) \operatorname{tr}\left(s_{m} x_{1}\right) \operatorname{tr}\left(t_{m-1} y_{1}\right) \cdots \operatorname{tr}\left(s_{m-1+1} x_{i}\right) \operatorname{tr}\left(t_{m-1} y_{i}\right) \\
\cdots \operatorname{tr}\left(t_{1} y_{n-1}\right) \operatorname{tr}\left(s_{1} x_{n}\right) \operatorname{tr}\left(y_{n}\right)=0,
\end{array}
$$

in case $m=n$;

$$
f(b a)=\operatorname{tr}\left(t_{m}\right) \operatorname{tr}\left(s_{m} x_{1}\right) \operatorname{tr}\left(t_{m-1} y_{1}\right) \cdots \operatorname{tr}\left(s_{2} x_{2}\right) \operatorname{tr}\left(t_{1} y_{n}\right) \operatorname{tr}\left(s_{1}\right)=0
$$

in case $m=n+1$. In all other cases, $b a$ is clearly a sum of irreducible monomials all of length at least two, hence $f(b a)=0$.

(iv) If both $x_{1}$ and $t_{m}$ are scalars, then

$$
b a=\operatorname{tr}\left(x_{1}\right) \operatorname{tr}\left(t_{m}\right) s_{1} t_{1} \cdots s_{m} y_{1} \cdots x_{n} y_{n}
$$

is an irreducible monomial of length at least two in case $m \neq 1$ or $n \neq 1$. Hence $f(b a)=0$. In cases $n=1$ and $m=1$,

$$
b a=\operatorname{tr}\left(x_{1}\right) \operatorname{tr}\left(t_{1}\right) s_{1} y_{1}, \quad \text { and } \quad f(b a)=0,
$$

since $s_{1}$ is not a scalar. Therefore $\operatorname{tr}(b a)=\operatorname{tr}(a b)$ in Case 1 .

Case 2. $s_{1}$ is a scalar, but $y_{n}$ is not. In case $m=n$,

$$
f(a b)=\operatorname{tr}\left(s_{1}\right) \operatorname{tr}\left(y_{n} t_{1}\right) \operatorname{tr}\left(x_{n} s_{2}\right)
$$

$$
\cdots \operatorname{tr}\left(y_{n-i+1} t_{i}\right) \operatorname{tr}\left(x_{n-i+1} s_{i+1}\right) \cdots \operatorname{tr}\left(y_{1} t_{m}\right) \operatorname{tr}\left(x_{1}\right)
$$

$$
f(a b)=\operatorname{tr}\left(s_{1}\right) \operatorname{tr}\left(y_{n} t_{1}\right) \operatorname{tr}\left(x_{n} s_{2}\right) \cdots \operatorname{tr}\left(y_{1} t_{m-1}\right) \operatorname{tr}\left(x_{1} s_{m}\right) \operatorname{tr}\left(t_{m}\right)
$$


in case $m=n+1$. In all other cases, $a b$ is a sum of irreducible monomials all of length at least two, hence $f(a b)=0$.

(i) If neither $x_{1}$ nor $t_{m}$ is a scalar, then $f(a b)=0$ by (8) and (9); also $b a=s_{1} t_{1} \cdots s_{m} t_{m} x_{1} y_{1} \cdots x_{n} y_{n}$, hence $f(b a)=0$.

(ii) If $x_{1}$ is a scalar but $t_{m}$ is not, then

$$
f(b a)=\operatorname{tr}\left(x_{1}\right) \operatorname{tr}\left(t_{m} y_{1}\right) \operatorname{tr}\left(s_{m} x_{2}\right) \cdots \operatorname{tr}\left(t_{1} y_{n}\right) \operatorname{tr}\left(s_{1}\right)=f(a b)
$$

in case $m=n$. In case $m=n+1, f(a b)=0$; and $b a$ is a sum of irreducible monomials all of length at least two, hence $f(b a)=0$. In case $n=m+1$,

$$
f(b a)=\operatorname{tr}\left(x_{1}\right) \operatorname{tr}\left(t_{m} y_{1}\right) \operatorname{tr}\left(s_{m} x_{2}\right) \cdots \operatorname{tr}\left(t_{1} y_{n-1}\right) \operatorname{tr}\left(s_{1} x_{n}\right) \operatorname{tr}\left(y_{n}\right)=0 ;
$$

and $a b$ is a sum of irreducible monomials all of length at least two, hence $f(a b)$ $=0$.

(iii) Suppose $t_{m}$ is a scalar but $x_{1}$ is not. In case $m=n, f(a b)=0$ and

$$
f(b a)=\operatorname{tr}\left(t_{m}\right) \operatorname{tr}\left(s_{m} x_{1}\right) \operatorname{tr}\left(t_{m-1} y_{1}\right) \cdots \operatorname{tr}\left(s_{1} x_{n}\right) \operatorname{tr}\left(y_{n}\right)=0 .
$$

In case $m=n+1$,

$$
f(b a)=\operatorname{tr}\left(t_{m}\right) \operatorname{tr}\left(s_{m_{1}} x_{1}\right) \operatorname{tr}\left(t_{m-1} y_{1}\right) \cdots \operatorname{tr}\left(s_{2} x_{n}\right) \operatorname{tr}\left(t_{1} y_{n}\right) \operatorname{tr}\left(s_{1}\right)=f(a b) .
$$

In all other cases $b a$ is a sum of irreducible monomials all of length at least two, hence $f(b a)=0=f(a b)$.

(iv) If both $x_{1}$ and $t_{m}$ are scalars, then $b a=\operatorname{tr}\left(x_{1}\right) \operatorname{tr}\left(t_{m}\right) s_{1} t_{1} \cdots s_{m} y_{1}$ $\ldots x_{n} y_{n}$ is an irreducible monomial of length at least two in case $m \neq 1$ or $n \neq 1$. Hence $f(b a)=0$. In case $n=m=1, f(b a)=\operatorname{tr}\left(s_{1}\right)_{\operatorname{tr}}\left(t_{1} y_{1}\right) \operatorname{tr}\left(x_{1}\right)=f(a b)$. Hence, $f(b a)=f(a b)$ in Case 2 .

Case 3. $y_{n}$ is a scalar but $s_{1}$ is not. In case $m=n$,

$$
\begin{aligned}
& f(a b)=\operatorname{tr}\left(y_{n}\right) \operatorname{tr}\left(x_{n} s_{1}\right) \operatorname{tr}\left(y_{n-1} t_{1}\right) \cdots \operatorname{tr}\left(y_{1} t_{m-1}\right) \operatorname{tr}\left(x_{1} s_{m}\right) \operatorname{tr}\left(t_{m}\right) \\
& f(a b)=\operatorname{tr}\left(y_{n}\right) \operatorname{tr}\left(x_{n} s_{1}\right) \operatorname{tr}\left(y_{n-1} t_{1}\right) \cdots \operatorname{tr}\left(x_{2} s_{m}\right) \operatorname{tr}\left(y_{1} t_{m}\right) \operatorname{tr}\left(x_{1}\right)
\end{aligned}
$$

in case $n=m+1$. In all other cases $a b$ is a sum of irreducible monomials all of length at least two, hence $f(a b)=0$.

(i) If neither $x_{1}$ nor $t_{m}$ is a scalar, then $b a=s_{1} t_{1} \cdots s_{m} t_{m}, x_{1} y_{1} \cdots$ $x_{n} y_{n}$ and $f(b a)=0$. We also have $f(a b)=0$ in this case.

(ii) Suppose $x_{1}$ is a scalar but $t_{m}$ is not. In case $m=n, f(a b)=0$, and

$$
\begin{aligned}
& f(b a)=\operatorname{tr}\left(x_{1}\right) \operatorname{tr}\left(t_{m} y_{1}\right) \operatorname{tr}\left(s_{m} x_{2}\right) \cdots \operatorname{tr}\left(t_{1} y_{n}\right) \operatorname{tr}\left(s_{1}\right)=0 \\
& f(b a)=\operatorname{tr}\left(x_{1}\right) \operatorname{tr}\left(t_{m} y_{1}\right) \operatorname{tr}\left(s_{m} x_{2}\right) \cdots \operatorname{tr}\left(t_{1} y_{n}\right) \operatorname{tr}\left(s_{1} y_{n}\right) \operatorname{tr}\left(y_{n}\right)=f(a b),
\end{aligned}
$$


in case $n=m+1$. In all other cases $b a$ is a sum of irreducible monomials all of length at least two, hence $f(b a)=0=f(a b)$.

(iii) Suppose $t_{m}$ is a scalar but $x_{1}$ is not. In case $m=n$,

$$
\begin{aligned}
& f(b a)=\operatorname{tr}\left(t_{m}\right) \operatorname{tr}\left(s_{m} x_{1}\right) \operatorname{tr}\left(t_{m-1} y_{1}\right) \cdots \operatorname{tr}\left(s_{1} x_{n}\right) \operatorname{tr}\left(y_{n}\right)=f(a b) ; \\
& f(b a)=\operatorname{tr}\left(t_{m}\right) \operatorname{tr}\left(s_{m} x_{1}\right) \cdots \operatorname{tr}\left(t_{1} y_{n}\right) \operatorname{tr}\left(s_{1}\right)=0,
\end{aligned}
$$

in case $m=n+1$ as $f(a b)=0$ also. In all other cases $b a$ is a sum of irreducible monomials all of length at least two. Hence, $f(b a)=0$ as $f(a b)=0$ too.

(iv) Suppose both $t_{m}$ and $x_{1}$ are scalars. Then $b a=\operatorname{tr}\left(x_{1}\right) \operatorname{tr}\left(t_{m}\right)_{s_{1} t_{1}}$ $\cdots s_{m} y_{1} \cdots x_{n} y_{n}$ is an irreducible monomial of length at least two in case $n \neq 1$ or $m \neq 1$; hence $f(b a)=0$, and in case $n=m=1, f(b a)=0$ since $\operatorname{tr}\left(s_{1}\right)=0$. By (10), in case $n=m, f(a b)=0$, since $\operatorname{tr}\left(x_{1} s_{m}\right)=\operatorname{tr}\left(x_{1}\right)_{\operatorname{tr}}\left(s_{m}\right)=0$. By (11), in case $n=m+1, f(a b)=0$, since $\operatorname{tr}\left(y_{1} t_{m}\right)=\operatorname{tr}\left(t_{m}\right) \operatorname{tr}\left(y_{1}\right)=0$, and $f(a b)=0$ in all other cases. Therefore, $f(a b)=f(b a)$ in Case 3 .

Case 4. Both $y_{n}$ and $s_{1}$ are scalars. Then $a b=\operatorname{tr}\left(s_{1}\right) \operatorname{tr}\left(y_{m}\right) x_{1} y_{1} \cdots x_{n} t_{1} \cdots$ $s_{m}{ }_{m}$. Hence $f(a b)=0$ if $n \neq 1$ or $m \neq 1$, and

$$
f(a b)=\operatorname{tr}\left(s_{1}\right) \operatorname{tr}\left(y_{1}\right) \operatorname{tr}\left(x_{1}\right) \operatorname{tr}\left(t_{1}\right) \quad \text { if } n=1 \text { and } m=1 .
$$

0 by (12).

(i) If neither $x_{1}$ nor $t_{m}$ is a scalar, then by (1) $f(b a)=0$ and $f(a b)=$

(ii) If $x_{1}$ is a scalar but $t_{m}$ is not, then $f(a b)=0$. In case $m=n$, by (2), $f(b a)=0$ since $\operatorname{tr}\left(t_{1} y_{n}\right)=\operatorname{tr}\left(t_{1}\right) \operatorname{tr}\left(y_{n}\right)=0$. In case $n=m+1$, by $(3), f(b a)=$ 0 since $\operatorname{tr}\left(s_{1} x_{n}\right)=\operatorname{tr}\left(s_{1}\right) \operatorname{tr}\left(x_{n}\right)=0$. And $f(b a)=0$ in all other cases as in Case 1 (ii).

(iii) If $t_{m}$ is a scalar but $x_{1}$ is not, then $\operatorname{tr}(a b)=0$. In case $m=n$, by (4), $f(b a)=0$ since $\operatorname{tr}\left(s_{1} x_{n}\right)=\operatorname{tr}\left(s_{1}\right) \operatorname{tr}\left(x_{n}\right)=0$. In case $m=n+1$, by $(5), f(b a)=$ 0 since $\operatorname{tr}\left(t_{1} y_{n}\right)=\operatorname{tr}\left(t_{1}\right) \operatorname{tr}\left(y_{n}\right)=0$. And $f(b a)=0$ in all other cases as in Case 1 (ii).

(iv) Suppose both $x_{1}$ and $t_{m}$ are scalars. Then $f(a b)=0=f(b a)$ if $m \neq$ 1 or $n \neq 1$; and $f(b a)=\operatorname{tr}\left(s_{1}\right) \operatorname{tr}\left(t_{1}\right) \operatorname{tr}\left(x_{1}\right) \operatorname{tr}\left(y_{1}\right)=f(a b)$ if $n=1$ and $m=1$. Therefore, $f(a b)=f(b a)$ in Case 4. This completes the proof for part (a).

Proof of (b). Let $a=\sum_{k=1}^{m} \sum_{k=1}^{n_{k}} x_{1, h} y_{1, b} \cdots x_{k, b} y_{k, b}$ be a nonzero element in $\bar{A}$, where each $x_{1, b} y_{1, b} \cdots x_{k, h} y_{k, b}$ in the summation is an irreducible monomial. Let $E$ be a maximal linearly independent finite subset containing 1, of the set $\left\{x_{k, b} \mid b=1, \ldots, n_{k}, k=1, \cdots, m\right\} \cup\{1\} . E$ is a finite linearly independent subset of $A_{1}$. Apply the Gram-Schmidt orthogonalization process to $E$ with respect to the inner product $\left(x \mid x^{\prime}\right)=\left(x \xi_{1} \mid x^{\prime} \xi_{1}\right)$ defined on $\Lambda_{1}$, we obtain an orthonormal set $F=\left\{a_{1}, a_{2}, \ldots, a_{n}\right\}$ in $\Lambda_{1}$, where we specifically design that $a_{1}=1$. Now let $O_{1}=\left\{a_{b}\right\}_{b \in N_{1}}$, where the index set $N_{1}$ is a subset of the set of all natural 
numbers containing $\{1, \ldots, n\}$, be a family of elements in $A_{1}$ which is maximal with respect to the following properties: (i) $F \subset O_{1}$; (ii) $\left(a_{b} \xi_{1} \mid a_{k} \xi_{1}\right)=0$ if $b \neq k$, and $\left(a_{b 1} \mid a_{k 1}\right)=1$ for $b=k \in N_{1}$. Let a subset $O_{2}=\left\{b_{k}\right\}_{k \in N_{2}}$ of $A_{2}$ be constructed similarly from the set $\left\{y_{k, b} \mid b=1, \ldots, n_{k}, k=1, \ldots, m\right\}$. Let $a=\left\{i_{1}, \ldots, i_{n}\right\}$ be an ordered set of $n$ elements from $N_{1}$ such that $i_{l} \neq 1$, if $l>1$, and let $\alpha^{\prime}=\left\{i_{1}^{\prime}\right.$, $\left.\cdots, i_{n}^{\prime}\right\}$ be an ordered set of $n$ elements from $N_{2}$ such that $i_{l} \neq 1$ if $l<n$. Let $S$ be the family of all such couples $\left(\alpha, \alpha^{\prime}\right)$ of ordered sets with elements from $N_{1}$ $\cup N_{2}$. For notational simplicity, we shall write $a$ for $\left(a, a^{\prime}\right)$ and $i_{k}$ for $i_{k}^{\prime}$.

Let $e_{\alpha}=e_{\left(a, a^{\prime}\right)}=a_{i_{1}} b_{i_{1}} \cdots a_{i_{n}} b_{i_{n}}, \alpha \in S$. We shall show that $f\left(e_{\alpha}^{*} e_{\alpha}\right)=1$ and $f\left(e_{a}^{*} e_{\beta}\right)=0$, where $e_{\beta}=a_{j_{1}} b_{j_{1}} \cdots a_{j_{m}} b_{j_{m}}, \alpha \neq \beta, \alpha, \beta \in S$.

Case 1. $a_{i_{1}}=b_{i_{n}}=1$. Then $e_{a}^{*}=a_{i_{n}}^{*} b_{i_{n-1}}^{*} \cdots a_{i_{2}}^{*} b_{i_{1}}^{*}$, and

$$
\begin{aligned}
f\left(e_{a}^{*} e_{\alpha}\right) & =f\left(\overline{\left.a_{i_{n}}^{*} b_{i_{n-1}}^{*} \cdots a_{i_{2}}^{*}\left(b_{i_{1} b_{i_{1}}}^{*}\right) a_{i_{2} b_{i_{2}}} \cdots a_{i_{n}} 1\right)}\right. \\
& =\operatorname{tr}\left(b_{i_{1}}^{*} b_{i_{1}}\right) f \overline{\left(a_{i_{n}}^{*} b_{i_{n-1}}^{*} \cdots\left(a_{i_{2}}^{*} a_{i_{2}}\right) b_{i_{2}} \cdots a_{i_{n}} 1\right)} \\
& =\left\|b_{i_{1}} \xi_{2}\right\|^{2}\left\|a_{i_{2}} \xi_{1}\right\|^{2} \cdots\left\|a_{i_{n}} \xi_{1}\right\|^{2}=1
\end{aligned}
$$

(i) Suppose $a_{j_{1}}=1$. If $n=m=1$, then $b_{j_{1}} \neq 1$ since $\alpha \neq \beta$. Hence $f\left(e_{a}^{*} e_{\beta}\right)=\operatorname{tr}\left(1 \cdot b_{j_{1}}\right)=0$. If $n=m>1$, then

$$
\begin{aligned}
f\left(e_{a}^{*} e_{\beta}\right) & =f \overline{\left(a_{i_{n}}^{*} b_{i_{n-1}}^{*} \cdots a_{i_{2}}^{*}\left(b_{i_{1}}^{*} b_{j_{1}}\right) a_{j_{2} b_{j_{2}} \cdots a_{j_{m}} b_{j_{m}}}\right)} \\
& =\operatorname{tr}\left(b_{i_{1}}^{*} b_{j_{1}}\right) \operatorname{tr}\left(a_{i_{2}}^{*} a_{j_{2}}\right) \cdots \operatorname{tr}\left(a_{i_{n}}^{*} a_{j_{m}}\right) \operatorname{tr}\left(b_{j_{m}}\right) .
\end{aligned}
$$

Since $O_{1}$ and $O_{2}$ are orthornormal sets in the inner products defined by traces, either one of $\left\{\operatorname{tr}\left(b_{i_{k}}^{*} b_{j_{k}}\right), \operatorname{tr}\left(a_{i_{k}}^{*} a_{j_{k}}\right)\right\}$ is zero or $\operatorname{tr}\left(b_{j_{m}}\right)=0$. Hence $f\left(e_{a}^{*} e_{\beta}\right)=0$. If $n=m+1$, then

$$
f\left(e_{a}^{*} e_{\beta}\right)=\operatorname{tr}\left(b_{i_{1}}^{*} b_{j_{1}}\right) \operatorname{tr}\left(a_{i_{2}}^{*} a_{j_{2}}\right) \cdots \operatorname{tr}\left(a_{i_{n}}^{*} a_{j_{m}}\right) \operatorname{tr}\left(b_{i_{m}}^{*} b_{j_{m}}\right) \operatorname{tr}\left(a_{i_{n}}^{*}\right)=0
$$

since $\operatorname{tr}\left(a_{i_{n}}\right)=0$. In all other cases $e_{\alpha}^{*} e_{\beta}$ is a sum of irreducible monomials all of length at least two, hence $f\left(e_{a}^{*} e_{\beta}\right)=0$.

$f\left(e_{a}^{*} e_{\beta}\right)=0$.

(ii) If $a_{i_{1}} \neq 1$, then $e_{a}^{*} e_{\beta}=a_{i_{n}}^{*} b_{i_{n-1}}^{*} \cdots a_{i_{2}}^{*} b_{i_{2}}^{*} a_{j_{1}} b_{j_{1}} \cdots a_{i_{m}} b_{i_{m}}$. Hence

Case 2. $a_{i_{1}}=1, b_{i_{n}}=1$. Then $e_{a}^{*}=1 b_{i_{n}}^{*} a_{i_{n}}^{*} b_{i_{n-1}}^{*} \cdots a_{i_{2}}^{*} b_{i_{1}}^{*}$, and

$$
\begin{aligned}
f\left(e_{a}^{*} e_{a}\right) & =f\left(1 b_{i_{n}}^{*} a_{i_{n}}^{*} b_{i_{n-1}}^{*} \cdots a_{i_{n}}^{*}\left(b_{i_{1}}^{*} b_{i_{1}}\right) a_{i_{2}} b_{i_{2}} \cdots a_{i_{n}} b_{i_{n}}\right) \\
& =\left\|b_{i_{1}} \xi_{2}\right\|^{2}\left\|a_{i_{2}} \xi_{1}\right\|^{2} \cdots\left\|a_{i_{n}} \xi_{1}\right\|^{2}=1 .
\end{aligned}
$$


(i) Suppose $a_{j_{1}}=1$. If $n=m$,

$$
f\left(e_{a}^{*} e_{\beta}\right)=\operatorname{tr}\left(b_{i_{1}}^{*} b_{j_{1}}\right) \operatorname{tr}\left(a_{i_{2}}^{*} a_{j_{2}}\right) \cdots \operatorname{tr}\left(b_{i_{n}}^{*} b_{j_{n}}\right)=0
$$

since $a \neq \beta$, one of the factors must be zero as $O_{1}$ and $O_{2}$ are orthonormal sets. If $n \neq m, e_{\alpha}^{*} e_{\beta}$ is a sum of irreducible monomials all of length at least two. Hence $f\left(e_{a}^{*} e_{\beta}\right)=0$.

Case 3. $b_{i_{n}}=1, a_{i_{j}} \neq 1$. Then $e_{a}^{*}=a_{i_{n}}^{*} b_{i_{n}}^{*} \cdots a_{i_{2}}^{*} b_{i_{1}}^{*} a_{i_{1}}^{*} 1$, and

$$
f\left(e_{a}^{*} e_{a}\right)=\operatorname{tr}\left(a_{i_{1}}^{*} a_{i_{1}}\right) \operatorname{tr}\left(b_{i_{1}}^{*} b_{i_{1}}\right) \cdots \operatorname{tr}\left(a_{i_{n}}^{*} a_{i_{n}}\right)=1 \text {. }
$$

$f\left(e_{a}^{*} e_{\beta}\right)=0$.

(i) If $a_{i_{1}}=1$, then $e_{\alpha}^{*} e_{\beta}=a_{i_{n}}^{*} b_{i_{n-1}}^{*} \cdots a_{i_{2}}^{*} b_{i_{1}}^{*} a_{i_{1}}^{*} b_{i_{1}} a_{i_{m}} b_{i_{m}}$, and

(ii) Suppose $a_{j_{1}} \neq 1$. If $n=m$, then

$$
f\left(e_{a}^{*} e_{\beta}\right)=\operatorname{tr}\left(a_{i_{1}}^{*} a_{j_{1}}\right) \operatorname{tr}\left(b_{i_{1}}^{*} b_{j_{1}}\right) \cdots \operatorname{tr}\left(a_{i_{n}}^{*} a_{j_{n}}\right) \operatorname{tr}\left(b_{j_{m}}\right)=0
$$

since $\alpha \neq \beta$, one of the factors above must be zero. If $n=m+1$, then

$$
f\left(e_{a}^{*} e_{\beta}\right)=\operatorname{tr}\left(a_{i_{1}}^{*} a_{j_{1}}\right) \operatorname{tr}\left(b_{i_{1}}^{*} b_{j_{1}}\right) \cdots \operatorname{tr}\left(a_{i_{n}}^{*} a_{i_{m}}\right) \operatorname{tr}\left(a_{i_{n}}^{*}\right)=0,
$$

since $a_{i_{n}} \neq 1$. In all other cases, $e_{a}^{*} e_{\beta}$ is a sum of irreducible monomials all of length at least two, hence $f\left(e_{a}^{*} c_{\beta}\right)=0$.

Case 4. $a_{i_{1}} \neq 1, b_{i_{n}} \neq 1$. Then $e_{a}^{*}=1 b_{i_{n}}^{*} a_{i_{n}}^{*} b_{i_{n}}^{*} \cdots a_{i_{2}}^{*} b_{i_{1}}^{*} a_{i_{1}}^{*} 1$, and

$$
f\left(e_{a}^{*} e_{a}\right)=\operatorname{tr}\left(a_{i_{1}}^{*} a_{i_{1}}\right) \operatorname{tr}\left(b_{i_{1}}^{*} b_{i_{1}}\right) \cdots \operatorname{tr}\left(b_{i_{n}}^{*} b_{i_{n}}\right)=1 \text {. }
$$

$f\left(e_{a}^{*} e_{\beta}\right)=0$.

(i) If $a_{i_{1}}=1$, then $e_{a}^{*} e_{\beta}=1 b_{i_{n}}^{*} \cdots a_{i_{2}}^{*} b_{i_{1}}^{*} a_{i_{1}}^{*} b_{i_{1}} \cdots a_{i_{m}} b_{i_{m}}$. Hence

(ii) Suppose $a_{j_{1}} \neq 1$. If $n=m$, then

$$
f\left(e_{a}^{*} e_{\beta}\right)=\operatorname{tr}\left(a_{i_{1}}^{*} a_{j_{1}}\right) \operatorname{tr}\left(b_{i_{1}}^{*} b_{j_{1}}\right) \cdots \operatorname{tr}\left(b_{i_{n}}^{*} b_{j_{n}}\right)=0
$$

since $\alpha \neq \beta$, so one of the factors above must be zero. If $m=n+1$,

$$
f\left(e_{a}^{*} e_{\beta}\right)=\operatorname{tr}\left(a_{i_{1}}^{*} a_{j_{1}}\right) \cdots \operatorname{tr}\left(b_{i_{n}}^{*} b_{j_{n}}\right) f\left(a_{j_{m}} b_{j_{m}}\right)=0
$$

since $a_{j_{m}} \neq 1$. In all other cases, $e_{\alpha}^{*} c_{\beta}$ is a sum of irreducible monomials all of length at least two, hence $f\left(e_{\alpha}^{*} c_{\beta}\right)=0$. We have proven that $f\left(e_{a}^{*} e_{a}\right)=1$ and $f\left(e_{a}^{*} e_{\beta}\right)=0$ if $a \neq \beta$ for all $a, \beta \in S$.

Now, let $a=\Sigma_{a \in S^{\prime} c_{a} c_{a}}$, where $c_{a}$ 's are nonzero complex numbers and $S^{\prime}$ is a finite subset of $S$. Hence

$$
f\left(a^{*} a\right)=f\left(\sum_{a, \beta \in S^{\prime}} \bar{c}_{a} c_{\beta} e_{\alpha}^{*} e_{\beta}\right)=\sum_{a \in S^{\prime}}\left|c_{\alpha}\right|^{2}>0
$$

for any $a \in \bar{A}$. 
Proof of (c). For $e_{a} \neq 1$, we have $f\left(e_{a}\right)=\overline{f\left(e_{a}\right)}=0$ and $f(1)=\overline{f\left(1^{*}\right)}=1$. For a general element, $a=\Sigma c_{a} e_{a}$ in $A . f\left(a^{*}\right)=f\left(\Sigma_{a} c_{a} e_{a}^{*}\right)=\overline{f(a)}$. This completes the proof of the lemma.

Now, we define

$$
(a \mid b)=f\left(b^{*} a\right), \text { for } a, b \in \bar{A} .
$$

It is clear from the previous lemmata that this is an inner product on $\bar{A}$ satisfying the following properties:

(i) $(a \mid b)=\left(b^{*} \mid a^{*}\right)$ for $a, b \in \bar{A}$,

(ii) $(a b \mid d)=\left(b \mid a^{*} d\right)$ for $a, d, b \in \bar{A}$.

In order to prove that $\bar{A}$ is a Hilbert algebra, it only remains to show that the multiplication $a \mapsto b \cdot a$ is continuous on $\bar{A}$ in the norm topology defined by the inner product for each $b \in \bar{A}$.

Lemma 3. For each $b \in \bar{A}, a \mapsto b \cdot a$ is a bounded linear operator on $\bar{A}$ in norm defined bv the inner product.

Proof. Since any element $b$ in $\bar{A}$ is a finite sum of monomials, and any operator in a von Neumann algebra is a linear combination of four unitary operators in that von Neumann algebra (Dixmier [5, Proposition 3, p. 4]), every element in $b$ is a linear combination of elements of the form $u=\overline{u_{1} v_{1} \cdots u_{r} v_{r}}$, where $u_{i}, v_{i}$ are unitary operators in $A_{1}$ and $A_{2}$ respectively, $i=1, \ldots, r$. Hence we only need to show that $L_{u}: a \rightarrow u \cdot a$ is a unitary operator on $\bar{A}$.

Let $a$ be given as in Lemma 2. Let the orthonormal set $\left\{e_{a}\right\}_{a \in S}$ be constructed as in Lemma 2, and let $e_{\alpha}, e_{\beta}$ be given as in Lemma 2. Then

$$
\begin{aligned}
& \left(u e_{\alpha} \mid u e_{\beta}\right)=f\left(e_{\beta}^{*} u^{*} u^{*} e_{\alpha}\right) \\
& =\overline{\left(1 b_{j_{m}}^{*} a_{j_{m}}^{*} b_{j_{m}}^{*} \cdots a_{j_{2}}^{*} b_{j_{1}}^{*} a_{j_{1}}^{*} v_{r}^{*} u_{r}^{*} v_{r-1}^{*} \cdots u_{2}^{*} v_{1}^{*}\left(u_{1}^{*} u_{1}\right) v_{1} \cdots u_{r} v_{r} a_{i_{1}} b_{i_{1}} \cdots a_{i_{n} b_{n}}\right)} \\
& =f\left(\overline{\left.1 b_{j_{m}}^{*} a_{j_{m}}^{*} b_{j_{m}}^{*} \cdots a_{j_{2}}^{*} b_{j_{1}}^{*}\left(a_{j_{1}}^{*} a_{i_{1}}\right) b_{j_{1}} \cdots a_{i_{n} b_{n}}\right)}=\left(e_{a} \mid e_{\beta}\right) .\right.
\end{aligned}
$$

Hence,

$$
(u a \mid u a)=\left(\sum_{a \in S^{\prime}} c_{a} u e_{a} \mid \sum_{a \in S^{\prime}} c_{a} u e_{a}\right)=\sum_{a, \beta} c_{\alpha} \bar{c}_{\beta}\left(u e_{a} \mid u e_{\beta}\right)=(a \mid a) .
$$

This shows that $u$ is unitary on $A$, and completes the proof of the lemma.

It can be proved similarly that $R_{b}: a \mapsto a \cdot b$ is a bounded linear operator on $\bar{A}$. Since $\bar{A}$ has an identity, $\bar{A}$ is a Hilbert algebra (Dixmier [5, Chapter $1, \S 5]$ ). Let $H$ be the completion of $\bar{A}$ under the defined inner product. Let $O_{1}=\left\{a_{b}\right\}_{b} \in N_{1}$ and $O_{2}=\left\{b_{k}\right\}_{k \in N_{2}}$ be maximal orthonormal sets in $A_{1}$ and. $A_{2}$ respectively, and let $\left\{e_{a}\right\}_{a \in S}$ be the orthonormal set in $\bar{A}$ constructed from $O_{1}$ and $O_{2}$ as in Lemma 2. Then every element $x$ of $H$ can be represented as $\Sigma_{a \in S} c_{\alpha} e_{a}$, which may have infinite many coefficients $c_{a}$ being nonzero, such that $\|x\|^{2}=\Sigma_{a \in S}\left|c_{a}\right|^{2}<$ $+\infty$. The left multiplication $L_{b}: a \rightarrow b \cdot a$ by an element $b$ in $A$ can be extended 
to a bounded linear operator $L_{b}$ on $H$. This is also true for multiplication by elements of $A$ on the right. We call the von Neumann algebra on $H$ generated by all left multiplication operators $L_{b}, b \in \bar{A}, A_{1} * A_{2}$, the free product of $A_{1}$ and $A_{2}$. Let $\hat{A}$ be the subset of $H$ consisting of all elements $x$ with the property that there exists a constant $K(x)$ such that $\|x \cdot a\| \leq K(x)\|a\|$ for all $a \in \bar{A} \subset H$. Hence, for each $x \in A, a \mapsto x \cdot a$ can be extended to a bounded linear operator $L_{x}$ on $H . \psi$ : $x \leftrightarrow L_{x}$ is a one-to-one correspondence between $\hat{A}$ and $A_{1} * A_{2}$. For let $x=$ $\Sigma_{\alpha \in S} c_{\alpha} e_{\alpha} \in \hat{A}$, and let $x_{i}=\Sigma_{\alpha \in F_{i}} c_{\alpha} e_{\alpha}$, where $F_{i}$ is a finite subset of $S, i=1$, $2, \ldots$, such that $\left\|x-x_{i}\right\|=\Sigma_{\alpha \in S \backslash F_{i}}\left|c_{a}\right|^{2} \rightarrow 0$; then $x_{i} \in \bar{A}, i=1,2, \ldots, L_{x_{i}} \rightarrow$ $L_{x}$ strongly, i.e. $L_{x} \in A_{1} * A_{2}$. On the other hand, it can be shown that $\left\{L_{x} \mid x \in \hat{A}\right\}$ $=\left\{R_{a} \mid x \in \bar{A}\right\}^{\prime}$ is already a von Neumann algebra, i.e. $A_{1} * A_{2} \subset \psi(\hat{A})$. By the properties of a Hilbert algebra, we have

(i) $\lambda L_{x}+\mu L_{y}=L_{\lambda x+\mu y}$, for $x, y \in \hat{A}$, and complex numbers $\lambda, \mu$.

(ii) $L_{x} L_{y}=L_{x \cdot y}$, for $x, y \in \hat{A}$, where $x \cdot y$ is the extended multiplication.

(iii) $\left(L_{x}\right)^{*}=L_{x^{*}}$, for $x \in \hat{A}$, where ${ }^{*}$ is the ${ }^{*}$ operation of $\bar{A}$ extended to $\hat{A}$. For simplicity, we identify $L_{x}$ in $A_{1} * A_{2}$ with $x$ in $\hat{A}$, and write $x$ for $L_{x}$. $A_{1} * A_{2}$ is a finite von Neumann algebra with trace $\operatorname{tr}(a)=(a \mid 1)$, for $a \in A_{1} * A_{2}$.

3. Factors of type $\mathbf{I I}_{1}$. Let $A$ be a von Neumann algebra on Hilbert space $H$ with a separating cyclic vector $\xi$. A countable set $O=\left\{U_{\alpha}\right\}_{a \epsilon I}$ of unitary operators in $A$ is called an ortho-unitary basis provided (i) $\left(U_{a} \xi \mid U_{\beta} \xi\right)=0$ if $\alpha \neq \beta$; (ii) for all $a \in I, U_{a}^{*}=c_{r} U_{r}$ for some $U_{r} \in O$ and a complex number $c_{r}$ of modulus 1 , and for every pair $\alpha, \beta \in I, U_{\alpha} U_{\beta}=c_{\mu} U_{\mu}$ for some $U_{\mu} \in O$ and a complex number $c_{\mu}$ of modulus 1 ; (iii) the set of all linear combinations of elements from $O$ is weakly (hence strongly) dense in $A$. Hence, every element $T$ in $A$ can be expressed as $\Sigma_{a \in I} c_{a} U_{a}$ uniquely, where the series converges unconditionally in strong-operator topology. If $T \in A$ is such that $\left(T \xi \mid U_{\alpha} \xi\right)=0$ for all $\alpha \in I$, then $T=0$. In other words, $\left\{U_{a}\right\}_{a \in I}$ is a maximal orthogonal set in $A$.

Remark 1. Let $G$ be a discrete group, then the von Neumann algebra $\mathscr{Q}(G)$ on $l^{2}(G)$ with $\delta_{e}$ as a separating cyclic vector has an ortho-unitary basis $\left\{U_{g}\right\}_{g} \in G$, where $U_{g}$ is the unitary operator corresponding to the translation by $g$. Conditions (i) and (ii) are trivially satisfied, and $\mathbb{A}(G)$ is the von Neumann algebra generated by $\left\{U_{g}\right\}_{g \in G}$.

Remark 2. Let $M_{n}$ be $n \times n$ matrix algebra, i.e. the factor of type $\mathrm{I}_{n}, n=1$, $2, \ldots$. Then $M_{n}$ is isomorphic to the von Neumann algebra $M_{n}=B\left(H_{n}\right) \stackrel{n}{\otimes} \mathrm{C}$ on $H_{n} \otimes H_{n}$, where $H_{n}$ is the $n$-dimensional Hilbert space, $B\left(H_{n}{ }_{n}\right)$ is the algebra of all linear operators on $H_{n}$, with $\xi=\left(\xi_{1}, \ldots, \xi_{n^{2}}\right)$ as a separating cyclic vector, where $\xi_{1}=\xi_{2+n}=\xi_{3+2 n}=\cdots=\xi_{k+(k-1) n}=\cdots=\xi_{n^{2}}=1$ and all other $\xi_{i}=0$. Let $W=a \otimes I$, and $V=b \otimes I$, where $a=\left(a_{i j}\right), a_{n 1}=a_{12}=a_{23}=a_{(n-1) n}=1$ and all other $a_{i j}=0 \quad(i, j=1, \cdots, n)$, and $b=\left(b_{i j}\right), b_{i j}=0 \quad(i \neq j)$, and 
$b_{j j}=e^{2 \pi i(j-1) / n}(j=1, \ldots, n)$ are two operators on $H_{n}$. Let $U_{k j}=W^{k} V^{j}, k=$ $1, \cdots, n, j=1, \cdots, n$. We claim that $\left\{U_{k, j}\right\}_{k, j=1}^{n}$ is an ortho-unitary basis for $M_{n}$. Each $U_{k j}$ is clearly a unitary operator. If $k \neq k^{\prime}$, then obviously we have $\left(U_{k, j} \xi \mid U_{k^{\prime}, j^{\prime}} \xi\right)=0$; if $k=k^{\prime}$, but $j \neq j^{\prime}$, then

$$
\left(U_{k, j} \xi \mid U_{k^{\prime}, j^{\prime}} \xi\right)=1+e^{2 \pi i\left(j-j^{\prime}\right) n-1 / n}=0 .
$$

We note that $V W=e^{2 \pi(n-1) i / n} W V,\left(V^{j}\right)^{*}=V^{n-j},\left(W^{k}\right)^{*}=W^{n-k}, j, k=1,2, \ldots, n$. Hence $U_{k, j}^{*}=\left(V^{j}\right) *\left(W^{k}\right) *=V^{n-j} W^{n-k}=e^{2 \pi i(n-1)(n-j)(n-k) / n} U_{n-k, n-j}$, $U_{k, j} U_{k^{\prime}, j^{\prime}}=W^{k} V^{j} W^{k^{\prime}} V^{j^{\prime}}=e^{2 \pi i(n-1) k^{\prime} j} U_{k+k^{\prime}, j+j^{\prime}}, k, j=1,2, \ldots, n$. And as $M_{n}$ is a $n^{2}$-dimensional linear space, $\left\{U_{k, j}\right\}_{k, j=1}^{n}$ spans $M_{n}$. Therefore, $\left\{U_{k, j}\right\}_{k, j=1}^{n}$ is an ortho-unitary basis for $M_{n}$.

Remark 3. Let $A_{1}$ and $A_{2}$ be two von Neumann algebras with separating cyclic trace vectors $\xi_{1}$ and $\xi_{2}$. Let $O_{1}=\left\{a_{\beta}\right\}_{\beta \in I_{1}}$, and $O_{2}=\left\{b_{r}\right\}_{r \in I_{2}}$ be ortho-unitary bases for $A_{1}$ and $A_{2}$ respectively. Let $O=\left\{e_{a}\right\}_{a \in S}$ be the orthonormal set of operators in $A_{1} * A_{2}$ constructed from $O_{1}$ and $O_{2}$ as we did in Lemma 3. $e_{a}^{\text {'s }}$ are, in fact, unitary operators in $A_{1} * A_{2}$. Conditions (i) and (ii) of an ortho-unitary basis can easily be verified, and the linear combinations of $e_{\alpha}$ 's form a dense set in $A_{1} * A_{2}$. Hence, $O$ is an ortho-unitary basis for $A_{1} * A_{2}$.

Theorem 1. Let $A_{1}$ and $A_{2}$ be two von Neumann algebras with separating cyclic trace vectors $\xi_{1}$ and $\xi_{2}$ on Hilbert space $H_{1}$ and $H_{2}$ respectively, where the linear dimension of $A_{1}$ is at least two and that of $A_{2}$ is at least three. Suppose that $A_{1}$ and $A_{2}$ bave ortho-unitary bases $O_{1}$ and $O_{2}$ respectively. Then the free product $A_{1} * A_{2}$ of $A_{1}$ and $A_{2}$ is a factor of type $\mathrm{II}_{1}$.

Proof. Let $O=\left\{e_{\alpha}\right\}_{a \in S}$ be the ortho-unitary basis of $A_{1} * A_{2}$ constructed from $O_{1}$ and $O_{2}$. Let $T=\Sigma_{a \epsilon S} c_{a} e_{a}$ be an arbitrary element in the center of $A_{1}$ * $A_{2}$, where the $c_{a}$ 's are complex coefficients. Suppose that $T$ is not a scalar. By rearrangements, we can assume that $r=\left|c_{a_{1}}\right| \neq 0$ and $e_{a_{1}} \neq 1$. Let $e_{a_{1}}=a_{\beta_{1}} b_{\beta_{1}}$ $\cdots a_{\beta_{n}} b_{\beta_{n}}$, where $n \geq 2$ if $a_{\beta_{1}}=1$ and $b_{\beta_{n}}=1$. Let $p=\max \left\{\rho\left(e_{a}\right)|| c_{a} \mid \geq r\right\}$.

Case 1. $a_{\beta_{1}} \neq 1$ and $b_{\beta_{n}} \neq 1$. Let $a_{\beta_{0}}$ be a nonidentity element of $O_{1}$ and let $b_{\beta_{0}}$ be a nonidentity element of $O_{2}$ orthogonal to $b_{\beta_{n}}$. Let $U=a_{\beta_{0}} b_{\beta_{0}}$. Let $a_{\beta_{0}}^{*}$ $=c_{\beta_{0}}^{\prime} a_{r_{0}}, b_{\beta_{0}}^{*}=c_{\beta_{0}}^{\prime \prime} b_{r_{0}}$, where $\left|c_{\beta_{0}}^{\prime}\right|=1 .\left|c_{\beta_{0}}^{\prime \prime}\right|=1, a_{r_{0}} \in O_{1}, b_{r_{0}} \in O_{2}$. Then $U^{*}=c_{\beta_{0}} 1 b_{r_{0}} a_{r_{0}} 1$, where $c_{\beta_{0}}=c_{\beta_{0}}^{\prime} c_{\beta_{0}}^{\prime \prime}$. Let $b_{\beta_{n}} b_{r_{0}}=c_{r_{0}} b_{r_{1}}$, where $b_{r_{1}} \neq 1$ since $b_{r_{0}}$ is orthogonal to $b_{\beta_{n}^{-1}}^{-1}$. We have

$$
\begin{aligned}
U^{k} T\left(U^{*}\right)^{k}= & c_{r_{0}}\left(c_{\beta_{0}}\right)^{k} c_{a_{1}}\left(a_{\beta_{0}} b_{\beta_{0}}\right)^{k} a_{\beta_{1}} b_{\beta_{1}} \cdots a_{\beta_{n}} b_{r_{1}}\left(a_{r_{0}} b_{r_{0}}\right)^{k-1} a_{r_{0}} 1 \\
& +\sum_{a \in S, a \neq a_{1}} c_{\alpha} U^{k} e_{\alpha}\left(U^{*}\right)^{k} .
\end{aligned}
$$

The first term has a coefficient with absolute value $r$, and the length of the irre- 
ducible monomial is $2 k+n$. And the second term is orthogonal to the first term because the trace is unitary invariant. But $U^{k} T\left(U^{*}\right)^{k}=T$ for all $k=1,2, \ldots$, and when $k>1 / a(p-n)$, we arrived at a contradiction by the definition of $p$. Hence $c_{a_{1}}=0$.

Case 2. $a_{\beta_{1}}=1$ and $b_{\beta_{n}}=1$. Let $a_{\beta_{0}} \neq 1, a_{\beta_{0}} \in O_{1}$, and $b_{\beta_{0}}$ be a nonidentity of $O_{2}$ orthogonal to $b_{\beta_{1}}^{*}$. Let $U=a_{\beta_{0}} b_{\beta_{0}}$. Then $U^{*}=1 b_{\beta_{0}}^{*} a_{\beta_{0}}^{*} 1=c_{\beta_{0}} 1 b_{r_{0}} a_{r_{0}} 1$, where $\left|c_{\beta_{0}}\right|=1$, and $b_{r_{0}} \in O_{2}, a_{r_{0}} \in O_{1}$. Let $b_{\beta_{0}} b_{\beta_{1}}=c_{r_{1}} b_{r_{1}}$, where $\left|c_{r_{1}}\right|=1$ and $b_{r_{1}} \neq 1, b_{r_{1}} \in O_{2}$. We have

$$
\begin{aligned}
U^{k} T\left(U^{*}\right)^{k}= & c_{r_{1}} c_{a_{1}} c_{\beta_{0}}^{k}\left(a_{\beta_{0}} b_{\beta_{0}}\right)^{k-1} a_{\beta_{0}} b_{r_{1}} a_{\beta_{2}} b_{\beta_{2}} \cdots a_{\beta_{n}}\left(b_{r_{0}} a_{r_{0}}\right)^{k-1} a_{r_{0}} 1 \\
& +\sum_{a \in S, a \neq a_{1}} c_{a} U^{k} e_{a}\left(U^{*}\right)^{k}, \quad k=1,2, \cdots
\end{aligned}
$$

The first term has a coefficient with absolute value $r$, and the length of the irreducible monomial is $2 k+n-1$; the second term is orthogonal to the first term. But $U^{k} T\left(U^{*}\right)^{k}=T, k=1,2, \ldots$, and when $k>1 / 2(p-n+1)$, we have a contradiction by the definition of $p$. Hence $c_{a_{1}}=0$.

Case 3. $a_{\beta_{1}}=1$ and $b_{\beta_{n}} \neq 1$. Let $a_{\beta_{0}} \neq 1, b_{\beta_{0}} \neq 1, a_{\beta_{0}} \in O_{1}, b_{\beta_{0}} \in O_{2}$. Let $a_{\beta_{0}}^{*}=$ $c_{r_{0}}^{\prime} a_{r_{0}}, b_{\beta_{0}}^{*}=c_{r_{0}}^{\prime \prime} b_{r_{0}}$, where $\left|c_{r_{0}}^{\prime}\right|=\left|c_{r_{0}}^{\prime \prime}\right|=1, a_{r_{0}} \in O_{1}, b_{r_{0}} \in O_{2}$. Let $U=$ $1 b_{\beta_{0}} a_{\beta_{0}} 1$; hence $U^{*}=c_{r_{0}} a_{r_{0}} b_{r_{0}}$, where $c_{r_{0}}=c_{r_{0}}^{\prime} c_{r_{0}}^{\prime \prime}$. We have

$$
\begin{aligned}
& U^{k} T\left(U^{*}\right)^{k}=c_{a_{1} c_{r_{0}}}{ }^{1 b_{\beta_{0}}}\left(a_{\beta_{0}} b_{\beta_{0}}\right)^{k-1} a_{\beta_{0}} b_{\beta_{1}} a_{\beta_{2}} b_{\beta_{2}} \cdots a_{\beta_{n}} b_{\beta_{n}}\left(a_{r_{0}} b_{r_{0}}\right)^{k} \\
& +\sum_{a \in S, a \neq a} c_{a} U^{k} e_{a}\left(U^{*}\right)^{k}, \quad k=1,2, \cdots
\end{aligned}
$$

The first term has a coefficient with absolute value $r$ and the length of the irreducible monomial is $2 k+n$; the second term is orthogonal to the first term. As $U^{k} T\left(U^{*}\right)^{k}=T, k=1,2, \ldots$, when $k>1 / 2(p-n)$, we have a contradiction by the definition of $p$. Hence $c_{a_{1}}=0$.

Case 4. $a_{\beta_{1}} \neq 1$ and $b_{\beta_{n}}=1$. Let $a_{\beta_{0}}$ and $b_{\beta_{0}}$ be nonidentity elements of $O_{1}$ and $O_{2}$ respectively. Let $a_{\beta_{0}}^{*}=c_{r_{0}}^{\prime} a_{r_{0}}, b_{\beta_{0}}^{*}=c_{r_{0}}^{\prime \prime} b_{r_{0}}$, where $\left|c_{r_{0}}^{\prime}\right|=\left|c_{r_{0}}^{\prime \prime}\right|=1$, $a_{r_{0}} \in O_{1}, b_{r_{0}} \in O_{2}$. Let $U=a_{\beta_{0}} b_{\beta_{0}}$; hence $U^{*}=c_{r_{0}} 1 b_{r_{0}} a_{r_{0}} 1$. We have

$$
\begin{aligned}
U^{k} T\left(U^{*}\right)^{k}= & c_{a_{1}} c_{r_{0}}^{k}\left(a_{\beta_{0}} b_{\beta_{0}}\right)^{k} a_{\beta_{1}} b_{\beta_{1}} \cdots a_{\beta_{n} b_{0} b_{r_{0}}\left(a_{r_{0}} b^{k-1} a_{r_{0}} 1\right.} \\
& +\sum_{a \in S, a \neq a_{1}} c_{a} U^{k} e_{a}\left(U^{*}\right)^{k}, \quad k=1,2,3, \cdots
\end{aligned}
$$

By the same reason as in the previous cases, when we let $k \geq 1 / 2(p-n)$, the equality $U^{k} T\left(U^{*}\right)^{k}=T$ together with the definition of $p$ implies a contradiction. Hence $c_{a_{1}}=0$. 
This proves that if $T$ is in the center then $T$ is a scalar. Hence $A_{1} * A_{2}$ is a factor. Since $A_{1} * A_{2}$ is clearly an infinite-dimensional vector space, $A_{1} * A_{2}$ is a factor of type $\mathrm{II}_{1}$ since $A_{1} * A_{2}$ has a trace vector. This completes the proof.

Let $O=\left\{e_{\alpha}\right\}_{\alpha \in S}$ be the ortho-unitary bases for the von Neumann algebra $A_{1} *$ $A_{2}$, constructed from $O_{1}$ of $A_{1}$ and $O_{2}$ of $A_{2}$. We define a multiplication on $S$ by $\alpha \cdot \beta=r$, if $e_{\alpha} \cdot e_{\beta}=c_{r} e_{r}$, where $c_{r}$ is a complex number of modulus 1 . This multiplication is uniquely defined. For if $e_{\alpha} \cdot e_{\beta}=c_{\mu} e_{\mu}$ with $e_{\mu} \neq e_{r}$, then $1=\left(e_{\alpha} \cdot\right.$ $\left.e_{\beta} \mid e_{a} \cdot e_{\beta}\right)=c_{r} c_{\mu}\left(e_{r} \mid e_{\mu}\right)=0$ a contradiction. If $e_{\alpha} e_{\beta}=c_{r} e_{r}$, and $\left(e_{r} e_{\mu}\right)=c_{\lambda} e_{\lambda}$, where $\left|c_{r}\right|=\left|c_{\lambda}\right|=1$, then $(a \beta) r=\lambda$. Let $e_{\beta} e_{r}=c_{\mu} e_{\mu^{\prime}}$, and $e_{\alpha} e_{\mu^{\prime}}=c_{\lambda^{\prime}} e_{\lambda^{\prime}}$, where $\left|c_{\mu}\right|=\left|c_{\lambda}\right|=1$. Then $\alpha(\beta r)=\lambda^{\prime}$. But $c_{r} c_{\lambda} e_{\lambda}=c_{\lambda^{\prime}} c_{\mu} e_{\lambda^{\prime}}$, by the same argument as before, we have $e_{\lambda^{\prime}}=e_{\lambda}$ or $\lambda=\lambda^{\prime}$. If $e_{l}=1$, then $\iota$ is clearly the identity of $S$. Also, we have $\alpha^{-1}=\beta$ if $e_{\alpha}^{*}=c_{\beta} e_{\beta}$, where $\left|c_{\beta}\right|=1$. Hence $S$ is a group. Let $E^{\prime}$ be the subset of $O$ consisting of all $e_{\alpha}$ ending in $a_{\beta_{0}} 1$, where $a_{\beta_{0}} \in O_{1}$, $a_{\beta_{0}} \neq 1$. Let $F=\left\{\beta: e_{\beta} \in E\right\}$. Let $e_{r_{0}}=a_{\beta_{0}} 1, e_{r_{1}}=1 b_{\beta_{1}}, e_{r_{2}}=1 b_{\beta_{2}}$, where $b_{\beta_{1}}, b_{\beta_{2}} \in O_{2}, b_{\beta_{1}} \neq 1, b_{\beta_{2}} \neq 1$. We have (i) $F \cup r_{0} F r_{0}^{-1}=S \backslash\{c\}$. (ii) $F, r_{1} F r_{1}^{-1}$ and $r_{2} \mathrm{Fr}_{2}^{-1}$ are three disjoint subsets of $S \backslash\{\imath\}$. The following is a slight variation of Lemma 10 in Pukanszky [13]. The proof, which we omit, is exactly the same after replacing $f\left(g_{i} a g_{i}^{-1}\right)$ by $c_{a} f\left(g_{i} a g_{i}^{-1}\right)$, where $c_{a}$ is a complex number of modulus 1 .

Lemma 4. Let $S$ be a group with identity 1 . Suppose there exists a subset $F \subset S \backslash\{c\}$ and three elements in $S$ such that (i) $F \cup g_{1} F g_{1}^{-1}=S \backslash\{c\}$, (ii) the sets $F, g_{2} F g_{2}^{-1}, g_{2} F g_{3}^{-1}$ are pairwise subsets of $S \backslash\{c\}$. Let $f$ be a complex-valued function on $S$ such that $\Sigma_{a \in S}|f(a)|^{2}<+\infty$,

$$
\left(\sum_{a \in S}\left|c_{a} f\left(g_{i} a g_{i}^{-1}\right)-f(g)\right|^{2}\right)^{1 / 2}<\epsilon \quad(i=1,2,3) .
$$

Then $\left(\Sigma_{a \in S \backslash\{c\}}|f(a)|^{2}\right)^{1 / 2}<14 \epsilon$.

Theorem 2. Let $A_{1}$ and $A_{2}$ be two von Neumann algebras with separating cyclic trace vectors. Suppose $A_{1}$ and $A_{2}$ bave ortbo-unitary bases $O_{1}$ and $O_{2}$ respectively, and that the linear dimension of $A_{1}$ is at least two, that of $A_{2}$ at least three. Then, the free product $A_{1} * A_{2}$ is a factor of type $\mathrm{II}_{1}$ without property $\Gamma$ (Murray and von Neumann [11, Definition 6.1]).

Proof. It is proved in Theorem 1 that $A_{1} * A_{2}$ is a factor of type $\mathrm{II}_{1}$. Suppose $A_{1} * \Lambda_{2}$ has property $\Gamma$. Let $1 / 14>\epsilon>0$, and let $T_{i}=e_{r_{i}}, i=0,1,2$, be given, where $e_{r_{i}}\left(\epsilon O=\left\{e_{a}\right\}_{a \in S}\right.$, the ortho-unitary basis of $A_{1} * A_{2}$ constructed from $O_{1}$ and $\left.O_{2}\right), i=0,1,2$, are as described in the discussion preceded by Lemma 4 . Let $U=\Sigma_{r} \in S f(r) e$, be a unitary operator in $A_{1} * A_{2}$ such that 
(i) $\operatorname{tr}(U)=0$, or $f(\iota)=0$, where $e_{\iota}=1$,

(ii) $\left\|U^{*} T_{i} U-T_{i}\right\|_{2}=\left\|T_{i} U T_{i}^{*}-U\right\|_{2}<\epsilon, i=0,1,2$, where $\|\cdot\|_{2}$ is the trace norm of $A_{1} * A_{2}$.

$$
\text { Let } e_{r_{i}}^{*}=c_{r_{i} r_{i}^{-1}}^{\prime} e_{r_{i}^{-1}} e_{r}=c_{r}^{\prime} e_{r_{i}-1}, e_{r_{i} 1_{r}} \cdot e_{r_{i}}=c_{r}^{\prime \prime} e_{r_{i}{ }^{1} r_{r}}, i=0,1,2, r \in S, S \text { is }
$$
given the group structure as described in the paragraph immediately before Lemma $4, c_{r_{i}}^{\prime}, c_{r}^{\prime}, c_{r}^{\prime \prime}$ are all complex numbers of modulus 1 . We have $\Sigma_{r} \in S \backslash\{\ell\}|f(r)|^{2}=1$, and by (ii)

$$
\left\|\sum_{r \in S}\left(c_{r} f(r) e_{r_{i}^{-1} r r_{i}}-f(r) e_{r}\right)\right\|_{2}=\left\{\sum_{r \in S}\left|c_{r} f\left(r_{i} r r_{i}^{-1}\right)-f(r)\right|^{2}\right\}^{1 / 2}<\epsilon,
$$

$i=0,1,2$. Let $F$ be the subset of $S$ described in the paragraph immediately before Lemma 4. Then, by Lemma 4, we have $\Sigma_{r \in S \backslash\{\ell\}}|f(r)|^{2}<14 \epsilon<1$, a contradiction. This completes the proof of the theorem.

Remark 4. Let $G_{1}$ and $G_{2}$ be two discrete groups, and let $G_{1} * G_{2}$ be the free product of $G_{1}$ and $G_{2}$. Then $\mathbb{A}\left(G_{1} * G_{2}\right)=\mathbb{Q}\left(G_{1}\right) * \mathbb{A}\left(G_{2}\right)$. In fact, let $U_{g}$ be the unitary operator in $\mathbb{Q}(G)$ corresponding to the translation by $g$ for $g \in G_{i}$, $i=$ 1, 2. Then $O_{i}=\left\{U_{g}\right\}_{g} \in G_{i}$ is an ortho-unitary basis for $\mathbb{Q}\left(G_{i}\right), i=1,2$. The orthounitary basis $O=\left\{e_{a}\right\}_{a \in S}$ for $\mathfrak{Q}\left(G_{1}\right) * \mathfrak{Q}\left(G_{2}\right)$ constructed from $O_{1}$ and $O_{2}$ is just $\left\{U_{g}\right\}_{g} \in G_{1} * G_{2}$ because $e_{a}$ 's are in one-to-one correspondence with the reduced sequences among all words in the free product $G_{1} * G_{2}$ (Magnus, Karrass and Solitar [7, Theorem 4.1]). Hence $\mathbb{Q}\left(G_{1}\right) * \mathbb{Q}\left(G_{2}\right)=\mathbb{Q}\left(G_{1} * G_{2}\right)$. For example, $\phi_{2}=$ $Z * Z$, the free product of two integer groups. Hence, $\mathbb{Q}\left(\Phi_{2}\right)=\mathbb{Q}(Z) * \mathbb{Q}(Z)$, the free product of two infinite-dimensional abelian von Neumann algebras.

In the case of $\mathbb{Q}\left(\Phi_{2}\right)=\mathbb{Q}(Z) * \mathbb{Q}(Z)$, there are three known types of outer automorphisms: (i) Interchange the two isomorphic free factors, i.e. $\phi: a \leftrightarrow b$, where $a$ and $b$ are the two generators of $\Phi_{2}$. This is first pointed out by Kadison [5, p. 308, Example 15]. (ii) Map $a$ into $a b, b$ into $b$, and extend this map into an automorphism of $\mathfrak{Q}\left(\Phi_{2}\right)$. (iii) Any outer automorphism of $\mathbb{Q}(Z)$ can be extended to an outer automorphism of $\mathbb{Q}(Z) * \mathbb{Q}(Z)$. This is pointed out by Behncke [1]. Now, let $M_{2}$ be the $2 \times 2$ matrix algebra with a separating cyclic trace vector on a fourdimensional Hilbert space, and let $M_{3}$ be the $3 \times 3$ matrix algebra with a separating cyclic trace vector on a nice-dimensional Hilbert space. Let $M_{2} * M_{3}$ be the free product of $M_{2}$ and $M_{3} \cdot M_{2} * M_{2}$ is a factor of type $\mathrm{II}_{1}$ without property $\Gamma$ as we remarked before, $M_{2}$ and $M_{3}$ have ortho-unitary bases. We can see that the three known types of outer automorphisms do not occur in the case of $M_{2} * M_{3}$ for (i) $M_{2}$ and $M_{3}$ are nonisomorphic, so they cannot be interchanged by an isomorphism; (ii) if $a$ is a generator for $M_{2}, b$ a generator for $M_{3}, a b$ would generate an infinitedimensional algebra, hence cannot be isomorphic to $M_{2}$; (iii) all automorphisms of 
$M_{2}$ and $M_{3}$ are inner. We cannot determine all outer automorphisms of $M_{2} * M_{3}$ at present. It may turn out that $M_{2} * M_{3}$ is a factor of type $\mathrm{II}_{1}$ without any outer automorphism. $M_{2} * M_{3}$ is also a possible candidate for a factor of type $\mathrm{II}_{1}$ nonisomorphic to any factor of type $\mathrm{II}_{1}$ constructed from group construction.

\section{REFERENCES}

1. H. Behncke, Automorphisms of $\mathfrak{Q}\left(\Phi_{2}\right)$, notes.

2. R. Blattner, Automorphic group representations, Pacific J. Math. 8 (1958), 665677.

3. W. Ching, Non-isomorphic non-hyperfinite factors, Canad. J. Math. 21 (1969), 12931308. MR 40 \#7822.

4. - A continuum of non-isomorphic non-hyperfinite factors, Comm. Pure Appl. Math. 23 (1970), 921-937. MR 43 \#5314.

5. J. Dixmier, Les algèbres d'opérateurs dans l'espace hilbertien (Algèbres de von Neumann), Cahiers scientifiques, fasc. 25, Gauthier-Villars, Paris, 1957. MR 20 \#1234.

6. J. Dixmier and E. C. Lance, Deux nouveaux facteurs de type $\mathrm{II}_{1}$, Invent. Math. 7 (1969), 226-234. MR 40 \#1787.

7. W. Magnus, A. Karrass and D. Solitar, Combinatorial group theory: Presentations of groups in terms of generators and relations, Pure and Appl. Math., vol. 13, Interscience, New York, 1966. MR 34 \#7617.

8. D. McDuff, A countable infinity of $\mathrm{II}_{1}$ factors, Ann. of Math. (2) 90 (1969), 361371. MR $41 \# 840$.

9. - Uncountably many II ${ }_{1}$ factors, Ann. of Math. (2) 90 (1969), 372-377. MR $41 \# 4261$.

10. F. Murray and J. von Neumann, On rings of operators, Ann. of Math. (2) 37 (1936), 116-229.

11. F. J. Murray and J. von Neumann, On rings of operators IV, Ann. of Math. (2) 44 (1943), 716-808. MR 5, 101.

12. M. Nakamura and Z. Takeda, On some elementary properties of the crossed products of von Neumann algebras, Proc. Japan Acad. 34 (1958), 489-494. MR 21 \#6550.

13. L. Pukánszky, Some examples of factors, Publ. Math. Debrecen 4 (1956), 135156. MR 18, 323.

14. S. Sakai, A symptotically abelian II ${ }_{1}$-factors, Publ. Res. Inst. Math. Sci. Ser. A 4 (1968/69), 299-307. MR 40 \#1785.

15. - An uncountable number of $\mathrm{II}_{1}$ and $\mathrm{II}_{\infty}$ factors, J. Functional Analysis 5 (1970), 236-246. MR $41 \# 4262$.

16. J. T. Schwartz, Two finite, non-hyperfinite, non-isomorphic factors, Comm. Pure Appl. Math. 16 (1963), 19-26. MR 26 \#6812.

17. N. Suzuki, Crossed products of rings of operators, Tôhoku Math. J. (2) 11 (1959), 113-124. MR $21 \# 4363$. 
18. M. Takesaki, Tomita's theory of modular Hilbert algebras and its applications, Lecture Notes in Math., vol. 128, Springer-Verlag, Berlin and New York, 1970. MR 42 \#5061.

19. T. Turumaru, Crossed product of operator algebras, Tohoku Math. J. (2) 10 (1958), 355-365. MR 21 \#1550.

20. J. von Neumann, On infinite direct product, Compositio Math. 6 (1938), 1-77.

21. G. Zeller-Meier, Deux autres facteurs de type II 1 , Invent. Math. 7 (1969), 235242. MR $40 \# 1788$.

DEPARTMENT OF MATHEMATICS, FORDHAM UNIVERSITY, NEW YORK, NEW YORK 10458 